Portland State University

PDXScholar

\title{
Inhibition of Interleukin-1 Receptor-Associated Kinase- 1 is a Therapeutic Strategy for Acute Myeloid Leukemia Subtypes
}

Mona Mohammad Hosseini

Portland State University

Follow this and additional works at: https://pdxscholar.library.pdx.edu/honorstheses

Let us know how access to this document benefits you.

\section{Recommended Citation}

Hosseini, Mona Mohammad, "Inhibition of Interleukin-1 Receptor-Associated Kinase-1 is a Therapeutic Strategy for Acute Myeloid Leukemia Subtypes" (2018). University Honors Theses. Paper 561.

https://doi.org/10.15760/honors.568

This Thesis is brought to you for free and open access. It has been accepted for inclusion in University Honors Theses by an authorized administrator of PDXScholar. Please contact us if we can make this document more accessible: pdxscholar@pdx.edu. 


\title{
" Inhibition of interleukin-1 receptor-associated kinase-1 is a therapeutic
} strategy for acute myeloid leukemia subtypes "

\author{
by \\ Mona M.Hosseini \\ An undergraduate honors thesis submitted in partial fulfillment of the \\ requirements for the degree of \\ Bachelor of Science \\ in \\ University Honors \\ and \\ Biology with minor in Chemistry
}

Thesis Adviser

Dr. Anupriya Agarwal

Portland State University

2018 


\begin{abstract}
A major challenge in treating acute myeloid leukemia (AML) is the molecular heterogeneity of the disease. Previously we showed that interleukin-1 (IL-1), promotes the growth of AML cells in 70\% of tested primary AML samples. Increased secretion of IL-1 results in increased levels of IL-1 receptor-associated kinase (IRAK1), an essential mediator of innate immunity and inflammatory responses. In this study, we showed overexpression of IRAK1 and its activity in AML across various genetic subtypes, and showed that genetic knockdown of IRAK1 gene by using inducible short hairpin RNA (shRNA) in AML cell lines and primary samples as well as xenograft model showed decrease in viability of leukemic cells. To determine the efficiency of the kinase inhibitor pacritinib, computational modeling along with engineered mutations showed specificity of pacritinib in binding to IRAK1. In compare to quizartinib (a FLT3 inhibitor) and ruxolitinib (a JAK1/2 inhibitor), pacritinib showed a broad sensitivity across AML cell lines as well as primary samples in decreasing the cell viability by reducing IRAK1 phosphorylation. Pacritinib treatment significantly reduced AML progenitors in xenograft model as well. Our data showed important role of IRAK1 in AML survival and how inhibition of its activity might provide beneficial therapeutically outcomes among heterogeneous AML subtypes.
\end{abstract}


Introduction

\section{Introduction}

Cancer, a top leading cause of mortality, is a major public health problem in the United States accounting for one in 4 deaths. ${ }^{1}$ Among all types of blood cancers, acute myeloid leukemia (AML) is the most common leukemia among adults with incident of more than $80 \%$ in patients of above 18 years old. ${ }^{2}$ AML is characterized as a genetically heterogeneous disease causing the clonal expansion of myeloid progenitors in the bone marrow and peripheral blood. Standard-of-care cytotoxic chemotherapy has been the most common treatment for this disease despite the fact that more than one third of patients show resistance to the chemotherapy, and the majority of patients would be diagnosed with relapsed or refractory disease which causes patients to have poor prognosis. ${ }^{3}$ Although the main cause of relapse is not clear, factors such as the substantial molecular heterogeneity inherent to AML disease, the emergence of genetically heterogeneous sub-clones, and pro-survival signals from the microenvironment as well as tumorintrinsic feedback pathways might account for the hidden sources. ${ }^{3}$ Thus, understanding the molecular mechanisms underlying the disease reveals some complexity of it leading to development of effective targeted therapy.

Some targeted therapies for AML have been developed due to recent whole-genome sequencing and expression studies uncovering some of the important heterogeneity in the molecular abnormalities driving AML. For example, the discovery of the activating internal tandem duplication (ITD) mutations in the FMS-related tyrosine kinase 3 (FLT3), which is detected in 20-25\% of AML patients, has led to development of small inhibitors such as sorafenib and quizartinib. ${ }^{4}$ As another example, gene-expression studies showed that mutations in nucleophosmin (NPM1) are present in 35\% of patients with AML and resulted in proposing several small inhibitors and compounds such as all-trans retinoic acid (ATRA) which has been 
shown to improve the outcomes of chemotherapy for patients harboring this mutation. ${ }^{4,5}$ On the other hand, some mutations such as Janus kinase 2 (JAK2) are found rarely in AML, yet profound in other blood cancers such as myeloproliferative neoplasms. ${ }^{6}$ Although using such specific small inhibitors show initial suppression of disease, the rise of secondary mutations causes resistance to the treatment. For example, although quizartinib (potent inhibitor of FLT3) shows positive results in treatment of AML patients harboring FLT3-ITD mutations, resistance develops in a short time due to signaling events induced by the microenvironment. ${ }^{7}$ Such these events have revealed the importance of inflammatory pathways in cancer initiation, progression, and development of resistance. ${ }^{8}$

The pro-inflammatory cytokine interleukin-1 (IL-1) is shown to provide expansion signal for myeloid progenitors in $67 \%$ of AML patients while it suppresses the growth of normal progenitors. ${ }^{9}$ Increased secretion of IL-1 in bone marrow microenvironment leads to activation of IL-1 receptor-associated kinase (IRAK1) and p38MAPK (MAPK14) in AML cells. IRAK, a central player in inflammatory responses by regulating the expression of various inflammatory genes in immune cells, is composed of IRAK-1,-2,-3 and -4 , with only -1 and -4 being active serine/threonine kinases, and expressed in variety of human immune cell types. ${ }^{10}$ IRAK signaling can be initiated from Toll-like receptors (TLRs) or from the interleukin-1 family receptors (IL1R). ${ }^{10}$ Activation of IL-1R and TLR recruits myeloid differentiation primary response 88 (MYD88), resulting in activation of IRAK4 and IRAK1. ${ }^{10,11}$ MYD88 coordinates with IRAK family to function as a signaling adaptor protein that activates the TRAF6-mediated NF- $\mathrm{B}$ and p38MAPK signaling. ${ }^{11,12} \mathrm{NF}-\kappa \mathrm{B}$ pathway regulates immune and inflammatory responses and protects cells from undergoing apoptosis in response to cellular stress, and p38MAPK pathway is a key regulator of pro-inflammatory cytokines biosynthesis at the transcriptional and 
translational levels. ${ }^{13,14}$ Oncogenically, active MYD88 mutations result in activation of IRAK signaling and such mutations have been determined in diffuse large B-cell lymphoma (DLBCL). ${ }^{15}$ Suppression of IRAK1 signaling in Waldenström's Macroglobulinaemia (WM) showed promising results as a therapeutic approach. ${ }^{15}$ Additionally, overexpression of IRAK1 in myelodysplastic syndromes (MDS) and all subtypes of T-cell acute lymphoblastic leukemia (TALL) is determined, and inhibition of IRAK1 by small inhibitor or gene knock down causes reduced survival of leukemic cells. ${ }^{15-17}$ In context of AML, several studies report overexpression of IRAK1/4 in the cancerous cells ${ }^{18,19}$, establishing the pathway as a candidate target in hematopoietic malignancies and emphasizing the need for agents that directly inhibit IRAK1 activity.

A previous in vitro profiling of 439 kinases using HotSpot assay found pacritinib, small ATP-competitive mycrocyclic molecule, as potent inhibitor of IRAK1, CFS1R, FLT3 and JAK2 ${ }^{20-22}$ Pacritinib is in development as a treatment for myelofibrosis. ${ }^{23,24}$ To evaluate the specificity of pacritinib in binding to IRAK1, computational modeling was performed by Agarwal laboratory and 3 residues on IRAK1 protein were identified to be important for pacritinib binding: serine 295 (S295) forming a hydrogen bond with an oxygen in pacritinib, adjacent glycine 294 (G294), and aspartate 298 (D298) forming a salt bridge with tertiary ammonium in pacritinib (Figure 2A). Engineered mutations of IRAK1 to resemble CDK2 was made by changing S295 to aspartate (S295D), deletion of G294 ( $\Delta \mathrm{G} 294)$, and changing D298 to lysine (D298K) (Figure 2B). The mutations conferred pacritinib resistance on IRAK1 with the D298K substitution conferring significant loss of pacritinib sensitivity (Figure 2C, 2D), confirming pacritinib binding to IRAK1 and not CDK2.

Upon confirmation of pacritinib specificity, to compare the efficacy of pacritinib with 
quizartinib (a FLT3 inhibitor) and ruxolatinib (a JAK2 inhibitor), Agarwal laboratory performed drug sensitivity assays using AML cell lines and primary samples $(n=46)$ by culturing cells in their respective media for $72 \mathrm{hrs}$ with graded concentrations of inhibitors and measuring cell viability by colorimetric MTS-based cell viability assay. Using fitted probit curves, $\mathrm{IC}_{50}, \mathrm{IC}_{90}$, and AUC values were measured and plotted. In both AML cell lines and primary samples, pacritinib showed lower $\mathrm{IC}_{50}$ compared to quizartinib and ruxolitinib (Figure 3A-B, 4A). In addition, pacritinib showed a reachable $\mathrm{IC}_{90}$ in primary samples and smaller AUC while AUC is correlated with $\mathrm{IC}_{50}$ values (Figure 4B-D).

The experimental evidences showed pacritinib as potent inhibitor of IRAK1. During this study I showed the important role of IRAK1 in regulation AML cell survival using in vitro and in vivo evidences, suggesting a rational for clinical trial for this indication. 


\section{Methods and Materials}

Cell lines and primary samples: MOLM-14, CMK, GDM-1, HT-93, and U-937 were maintained in RPMI-1640 medium supplemented with 10\% FBS. THP-1 was maintained in RPMI-1640 medium supplemented with 15\% FBS. Culture media was also substituted with 2 mM L-glutamine, $100 \mathrm{U} / \mathrm{mL}$ penicillin and $100 \mu \mathrm{g} / \mathrm{mL}$ streptomycin. All cell lines were cultured at $37^{\circ} \mathrm{C}$ in $5 \% \mathrm{CO}_{2}$. Cell lines used in this study were not cultured for longer than 6 months from initial purchase or characterization. Mononuclear cells isolated from 46 primary patient samples diagnosed with AML were evaluated according to Institutional Review Board guidelines at Oregon Health \& Science University and cultured in RPMI-1640 supplemented with 10\% FBS, 2 $\mathrm{mM}$ L-glutamine, and $100 \mathrm{U} / \mathrm{mL}$ penicillin-streptomycin and $10^{-4} \mathrm{M} \beta$-mercaptoethanol.

shRNA-mediated knockdown: An inducible short hairpin RNA (shRNA) library targeting human IRAK1 was obtained from Cellecta, Inc (Mountain View, CA). Lentivirus was generated by transfecting 293FT cells using VSV-G envelope plasmid, 8.9 helper plasmid, and FuGENE-6 reagent. Lentivirus particles were concentrated by ultra-centrifuging the viral supernatant at 28,000 rpm for $2 \mathrm{hr}$ at $4^{\circ} \mathrm{C}$. Primary cells and cell lines were infected with concentrated virus particles in the presence of $5 \mu \mathrm{g} / \mathrm{mL}$ polybrene and $1 \mathrm{mM}$ HEPES and centrifuged at 2,500 rpm for 90 minutes at $30^{\circ} \mathrm{C}$. This was repeated at $24 \mathrm{hr}$. For stable expression of shRNA, cells were GFP-sorted after 48 hours of infection and maintained in puromycin. To induce shRNA expression, cells were treated with $1 \mu \mathrm{g} / \mathrm{ml}$ doxycycline, and the effect of gene knockdown was assessed by quantitative PCR, cell viability assay, and immunoblotting.

Immunoblotting: Upon treatment of AML cell lines or primary samples with inhibitors, cells were obtained and lysed using $1 \mathrm{X}$ lysis buffer purchased from Cell Signaling (Danvers, MA). Standard protocol was used to quantify protein concentration and extraction with SDS buffer and 
SDS-PAGE gel. Proteins were identified using the following antibodies as indicated: p-IRAK1 (12756), p-FLT3 (3464), p-ERK1/2 (9101), p-p38 (4631), p-STAT5 (9351) (Cell Signaling Technology); GAPDH (AM4300) (ThermoFisher); IRAK1 (Santa Cruz Biotechnology); and pIRAK1 (SAB4504246) and $\alpha$-tubulin (T6074) (Sigma Chemicals).

In vivo Xenograft studies: MOML-14 cells expressing doxycycline inducible IRAK1 shRNA GFP+ $\left(2 \times 10^{5}\right.$ cell per mouse $)$ were injected into the tail veins of 4-week old NOD-scid IL2R $\mathrm{R}^{\text {gnull }}$ (NSG) mice purchased from Jackson Laboratories. Mice were monitored by performing differential blood counts using Heska Vet ABC blood analyzer (scil Animal Care Company, Gurnee, IL) and determining GFP percentage in their peripheral blood using flow cytometry. Treatment was started 5 days after the initial injection of cells and after confirmation of equal engraftment in peripheral blood (>1\%). Pacritinib was suspended in vehicle consisting of $0.5 \%$ weight/volume methylcellulose $(4000 \mathrm{cP})$ and $0.1 \%$ volume/volume TWEEN® 80 , and mice were treated twice daily by oral gavage at the dose of $150 \mathrm{mg} / \mathrm{kg}$ using $100 \mu \mathrm{L}$ volume. Doxycycline was suspended in $1 \%$ sucrose at concentration of $5 \mathrm{mg} / \mathrm{mL}$, and mice were treated once a day by oral gavage using $100 \mu \mathrm{L}$ volume ( $500 \mu \mathrm{g} / \mathrm{mouse}$ ). Doxycycline arm of mice were also maintained at the chow containing $625 \mathrm{mg} / \mathrm{kg}$ doxycycline (Envigo, D11072801). Animals were sacrificed at day 18 post treatment. The sizes of their spleens and livers were measured, and the cells of spleen, bone marrow, and peripheral blood were utilized to quantitate the leukemia burden. The cells were stained for live/dead marker (Fixable Aqua dye, ThermoFisher), incubated at $37^{\circ} \mathrm{C}$ for 30 minutes, and stained for human cell markers using CD13 (PE) and CD33 (PE) cell surface antibodies and mouse pan hematopoietic cell surface marker using CD45 (APC-Cy7) (BD Pharmingen) antibody followed by $1 \mathrm{hr}$ incubation at room temperature. The 
Methods and Materials

expressions of cell surface markers were measured by flow cytometry and analyzed using

FlowJo software. 


\section{Results}

IRAK1 Knockdown has profound effect on growth of AML cells.

By performing immunoblot on AML primary samples and normal cells, we observed elevated expression and activity of IRAK1 in AML (supplemental Figure 1). Upon knocking down IRAK1 gene in AML cell lines (MOLM-14 and THP-1) and primary samples using doxycycline-inducible shRNA, we observed reduction of IRAK1 expression by $70 \%$ in two of the three tested hairpins, measured by quantitative PCR and confirmed by immunoblotting (Figure 1A, 1B). The IRAK1 knock down reduced cell viability by $80 \%$ in MOLM-14 (FLT3ITD positive) and 40\% in THP-1 (FLT3-ITD negative) cell line (Figure 1C). Also, IRAK1 knock down in primary samples reduced the viability up to $60 \%$ (Figure 1C). To enhance the results of knock down in primary samples, two hairpins were combined.

\section{IRAK1 inhibition by pacritinib suppresses growth of AML cell lines.}

As mentioned above, Agarwal laboratory showed a lower $\mathrm{IC}_{50}$ value for pacritinib inhibiting the growth of AML cell lines harboring different genetic abbreviations through drug sensitivity assays compared to quizartinib and ruxolitinib which were restricted in their capacity to inhibit growth across this panel (Figure 3A-B). The effect of IRAK1 inhibition on downstream signaling was determined through immunoblot analysis. In MOLM-14 cell line, all three inhibitors reduced phosphorylation of IRAK1 on residue T209 at various levels. Also, pacritinib and quizartinib, but not ruxolitinib, reduced phosphorylation of STAT5 and p38 and inhibited activation of FLT3-ITD (Figure 3C). Further, pacritinib inhibited IRAK1 activation in CSF1Rdependent cells GDM-1, JAK3 mutation-positive CMK-1, and MLL-AF9-positive THP1 cells (Figure 3C). In addition, pacritinib inhibited IRAK1 in PICALM-AF10-dependent U937 cells that showed resistance to the inhibitor previously (Figure 3C). When higher concentrations of 
quizartinib was tested in MOLM-14 cells, IRAK1 phosphorylation was not inhibited compared to pacritinib suggesting IRAK1 might not be the direct target of FLT3 (Supplemental Figure 2). Pacritinib has a potent sensitivity profile across a broad range of genetic subtypes in primary AML patient samples.

As mentioned in the introduction, Agarwal laboratory observed a significantly lower median $\mathrm{IC}_{50}$ compared with that for quizartinib and ruxolitinib when tested inhibitors against primary AML samples $(n=46)$. Through this assay, pacritinib showed a higher response rate $(85 \%$; $39 / 46)$ than that observed for either quizartinib $(54 \% ; 25 / 46)$ or ruxolitinib $(11 \% ; 5 / 46)$, where responsiveness was defined as an $\mathrm{IC}_{50}$ value $<500 \mathrm{nM}$. Notably, comparison of median $\mathrm{IC}_{90}$ values for pacritinib and quizartinib suggested that pacritinib treatment leads to an achievable IC 90 as well as smaller AUC (Figure 4A-D). Through probit mixed effects population curve modeling on AML samples, Agarwal laboratory observed that quizartinib was sensitive in inhibiting growth within samples harboring FLT3 mutations whereas pacritinib showed sensitivity for both samples with mutant FLT3 and wild type FLT3. Analysis through flow cytometry of primary samples treated with pacritinib for 72 hours showed significant reduction in CD34+ progenitors (supplemental Figure 3). The effect on IRAK1 inhibition on downstream signaling in AML primary samples was determined through immunoblotting in which pacritinib reduced IRAK1 and p38 phosphorylation (Figure 4B). In addition, pacritinib reduced IRAK1 phosphorylation in IL-1 and LPS stimulated cells.

Genetic knockdown of IRAK1 or treatment with pacritinib reduces the leukemia burden in AML xenograft model in vivo.

To evaluated the efficacy of genetic knockdown of IRAK1 and pacritinib treatment in vivo, we injected MOLM-14 cells transduced with doxycycline-inducible IRAK1 shRNA into NOD- 
scid IL2Rg ${ }^{\text {null }}$ (NSG) mice (Figure 5A). After confirmation of engraftment by measuring GFP+ percentage on peripheral blood through flow cytometry at day post injection, mice were treated with doxycycline, pacritinib or vehicle. Since MOLM-14 cell are GFP+ and express CD13 and CD33, we used GFP positivity and the expression of human CD13/CD33 to quantify the effect of treatment on the leukemia burden. We observed that both IRAK1 knock down and inhibition by pacritinib reduced leukemia burden from $\sim 76 \%$ to $\sim 50 \%$ and $\sim 42 \%$, respectively, in the bone marrow and $\sim 42 \%$ to $\sim 8 \%$ and $\sim 10 \%$, respectively, in the spleen (Figure 5B-D). Further, the host murine cells in the bone marrow and spleen showed increase measured by GFP- cells or murine $\mathrm{CD}_{4} 5^{+}$cells. We also observed a significant reduction in spleen and liver sizes (Figure 5E, 5F). In addition, slight reduction in white blood counts with no significant differences in platelets, hemoglobin, and hematocrit with IRAK1 knockdown or pacritinib treatment was observed (Supplemental Figure 4). Overall, these results showed IRAK1 knockdown or inhibition by pacritinib reduced leukemia burden in vivo. 


\section{Discussion}

Treatment of AML by inhibiting one of the many molecular events has shown the rapid emergence of resistance and secondary mutations. This has brought the attention to the bone marrow microenvironment-mediated signaling that might be responsible for such occurrences. Here we provide evidence that IRAK1 pathway, a central mediator of innate immunity and inflammatory responses, is a therapeutic target across a variety of genetic subtypes in AML. We showed that IRAK1 in constitutively active in AML cells by being constitutive phosphorylated in different genetic subtypes. Although IRAK1 is overexpressed in AML cells compared to healthy cells, no correlation was found between IRAK1 activation and AML subtypes suggesting the pathway is uniformly active.

Our data showed that genetic knockdown of IRAK1 reduces viability of leukemic cells in vitro and leukemia burden in vivo significantly. This prompted us to test the efficacy of pacritinib as potent inhibitor of IRAK1 introduced by a previous study on kinase profiling. Through computational modeling and engineered mutations, Agarwal laboratory showed the specificity of pacritinib in binding to IRAK1 and tested this inhibitor in comparison to a FLT3 inhibitor (quizartinib) and a JAK2 inhibitor (ruxolitinib). Among AML cell lines and 46 primary samples, pacritinib showed more sensitivity in reducing the viability of cells harboring different genetic mutations. Immunoblotting of different AML cell lines and primary samples showed inhibitory effect of IRAK1 on down-stream signaling.

In vivo experiment of inhibiting activity of IRAK1 showed significant reduction of leukemia burden using xenograft model. Interestingly, the effect of pacritinib was much higher than knockdown of IRAK1 gene alone, suggesting the ability of pacritinib in inhibiting different kinases. 
Discussion

Overall, we provide evidence that innate immunity pathway involving IRAK1 is important in AML pathobiology. We showed pacritinib is effective in inhibiting the activity of IRAK1. Together these results suggest that IRAK1 can serve as a therapeutic target across various genetic subtypes, and therefore further clinical exploration of IRAK1 as a target for intervention with pacritinib and newer more specific agents is justified in AML. 


\section{References}

1- Siegel R, Naishadham D, Jemal A. Cancer statistics, 2012. CA Cancer J Clin.62:10-29 (2012).

2- Thein MS, Ershler WB, Jemal A, Yates JW, Baer MR. "Outcome of Older Patients with Acute Myeloid Leukemia: An analysis of SEER Data over Three Decades." Cancer. 2013;119(15):10.1002/cncr.28129. doi:10.1002/cncr.28129.

3- Ramos NR, Mo CC, Karp JE, Hourigan CS. Current Approaches in the Treatment of Relapsed and Refractory Acute Myeloid Leukemia. Ustun C, Godley LA, eds. Journal of Clinical Medicine. 2015;4(4):665-695. doi:10.3390/jcm4040665.

4- Guenounou S, Delabesse E, Récher C. Sorafenib plus all-trans retinoic acid for AML patients with FLT3- ITD and NPM1 mutations. European Journal Of Haematology [serial online]. December 2014;93(6):533-536.

5- Verhaak R. G., Goudswaard C. S., van Putten W., Bijl M. A., Sanders M. A., Hugens W., Uitterlinden A. G., Erpelinck C. A., Delwel R., Löwenberg B., \& Valk P. J. Mutations in nucleophosmin (NPM1) in acute myeloid leukemia (AML): association with other gene abnormalities and previously established gene expression signatures and their favorable prognostic significance. Blood, 106(12), 3747-3754 (2005).

6- Rawlings JS, Rosler KM, Harrison DA. The JAK/STAT signaling pathway. Journal of cell science 117, 1281-1283 (2004).

7- Traer E, Martinez J, Javidi-Sharifi N, et al. FGF2 from marrow microenvironment promotes resistance to FLT3 inhibitors in acute myeloid leukemia. Cancer research. 2016;76(22):6471-6482. doi:10.1158/0008-5472.CAN-15-3569.

8- Giles, FJ, Krawczyk J, O'Dwyer M, Swords R, Freeman C. The role of inflammation in leukaemia. Advances in experimental medicine and biology 816, 335-360 (2014).

9- Carey A, Edwards DK, Eide CA, et al. Identification of interleukin-1 by functional screening as a key mediator of cellular expansion and disease progression in acute myeloid leukemia. Cell reports. 2017;18(13):3204-3218. doi:10.1016/j.celrep.2017.03.018.

10- Jain A, Kaczanowska S, Davila E. IL-1 Receptor-Associated Kinase Signaling and Its Role in Inflammation, Cancer Progression, and Therapy Resistance. Frontiers in Immunology. 2014;5:553. doi:10.3389/fimmu.2014.00553.

11- Ngo VN, Young RM, Schmitz R, et al. Oncogenically active MYD88 mutations in human lymphoma. Nature. 2011;470(7332):115-119. doi:10.1038/nature09671.

12- Li Z, Younger K, Gartenhaus R, et al. Inhibition of IRAK1/4 sensitizes T cell acute lymphoblastic leukemia to chemotherapies. The Journal of Clinical Investigation. 2015;125(3):1081-1097. doi:10.1172/JCI75821.

13- Yamamoto Y, Gaynor R.B. Role of the NF-kB Pathway in the Pathogenesis of Human Disease States. Current Molecular Medicine (2001).

14- Cuenda A., Rousseau S. p38 MAP-Kinases pathway regulation, function and role in human diseases. Molecular Cell Research (2007).

15- Rhyasen GW, Starczynowski DT. IRAK signalling in cancer. British Journal of Cancer. 2015;112(2):232-237. doi:10.1038/bjc.2014.513.

16- Rhyasen GW, Bolanos L, Fang J, et al. Targeting IRAK1 as a therapeutic approach for Myelodysplastic Syndrome. Cancer cell. 2013;24(1):90-104.

doi:10.1016/j.ccr.2013.05.006. 
17- Dussiau C, Trinquand A, Lhermitte L, et al. Targeting IRAK1 in T-Cell acute lymphoblastic leukemia. Oncotarget. 2015;6(22):18956-18965.

18- Rhyasen GW, Bolanos L, Starczynowski DT. Differential IRAK signaling in hematologic malignancies. Experimental hematology. 2013;41(12):1005-1007. doi:10.1016/j.exphem.2013.09.008.

19- Beverly LJ, Starczynowski DT. IRAK1: oncotarget in MDS and AML. Oncotarget. 2014;5(7):1699-1700.

20- Anastassiadis T, Deacon SW, Devarajan K, Ma H, Peterson JR. Comprehensive assay of kinase catalytic activity reveals features of kinase inhibitor selectivity. Nature biotechnology 2011 Oct 30; 29(11): 1039-1045.

21-Singer JW, Al-Fayoumi S, Ma H, Komrokji RS, Mesa R, Verstovsek S. Comprehensive kinase profile of pacritinib, a nonmyelosuppressive Janus kinase 2 inhibitor. Journal of Experimental Pharmacology. 2016;8:11-19. doi:10.2147/JEP.S110702.

22- Poulsen A, William A, Blanchard S, Lee A, Nagaraj H, Wang H, et al. Structure-based design of oxygen-linked macrocyclic kinase inhibitors: discovery of SB1518 and SB1578, potent inhibitors of Janus kinase 2 (JAK2) and Fms-like tyrosine kinase-3 (FLT3). Journal of computer-aided molecular design 2012 Apr; 26(4): 437-450.

23- Mascarenhas J, Hoffman R, Talpaz M, Gerds AT, Stein B, Gupta V, et al. Results of the Persist-2 Phase 3 Study of Pacritinib (PAC) Versus Best Available Therapy (BAT), Including Ruxolitinib (RUX), in Patients (pts) with Myelofibrosis (MF) and Platelet Counts $<100,000 / \mu 1$ Late Breaking Abstract at 58th American Society of Hematology (ASH) Annual Meeting and Exposition in San Diego; 2016.

24- Mesa RA, Vannucchi AM, Mead A, Egyed M, Szoke A, Suvorov A, et al. Pacritinib versus best available therapy for the treatment of myelofibrosis irrespective of baseline cytopenias (PERSIST-1): an international, randomised, phase 3 trial. The Lancet Haematology 2017 May; 4(5): e225-e236. 


\section{Figure Legends}

Figure 1. IRAK1 knockdown by shRNA reduces viability of AML cells. AML cell lines (MOLM-14, THP-1) and primary AML CD34-positive cells were infected with 3 independent inducible IRAK1 shRNAs (-1, -2, -3), and primary AML CD34-positive cells were infected with a combination of IRAK1 shRNA hairpins. After 48 hours of infection, cells were sorted for GFP positivity, and cells stably expressing IRAK1 shRNA were treated with doxycycline for $48 \mathrm{hr}$ and 6 days. The effect of knockdown was determined by measuring change in IRAK1 gene expression by qPCR (A), western blotting (B), and cell growth by MTS assay after 4 days of doxycycline treatment (C). Results shown are representative of 3 independent experiments.

Figure 2. Structural requirements for pacritinib binding in the IRAK1 kinase domain. Agarwal laboratory has determined the (A) molecular model of pacritinib-bound IRAK1. Ribbon diagram shows crystal structure-based homology model of IRAK1 kinase domain with pacritinib docked in the ATP-binding pocket. Key residues forming favorable interactions with the inhibitor are highlighted. (B) Kinase domain alignment of human IRAK family and kinase paralogs. Engineered IRAK1 mutations based on pacritinib-resistant CDK2 (DG294, S295D, and D298K) are indicated in the image in orange, green, and purple, respectively. (C) Representative immunoblot showing phosphorylation of wild-type versus mutant IRAK1 in heterologous expression system after short-term treatment with pacritinib. (D) Quantitative assessment of pacritinib inhibition of wild-type and mutant IRAK1. Percent inhibition is based on calculation of ratio of pIRAK1 to total (Flag) IRAK1 after densitometry of immunoblots. Results shown are representative of 3 independent experiments.

Figure 3. Pacritinib effectively targets AML cell lines harboring a broad range of genomic aberrations. Agarwal laboratory cultured AML cell lines in their respective culture 
media for 72 hours with graded concentrations of pacritinib, quizartinib, and ruxolitinib from 1.2 $\mathrm{nM}$ to $5000 \mathrm{nM}$ using 2-fold dilution curves. Cell viability was measured by colorimetric MTSbased cell viability assay. (A) Viability of representative cell lines. Each cell line was tested at least in triplicate, and data are represented as mean \pm SEM. (B) Scatter plot showing cell-based $\mathrm{IC}_{50}$ values for pacritinib, quizartinib, and ruxolitinib determined from cell-based assays with indicated AML cell lines by Agarwal laboratory. (C) Immunoblot analysis of lysates prepared from pacritinib-treated AML cell lines as indicated. Phosphorylation status of IRAK1, p38, STAT5, and ERK1/2 are shown before and after pacritinib treatment. GAPDH serves as a loading control. Immunoblot analysis of lysates prepared from U937, MOLM14, GDM-1, THP1, and CMK-1 cells treated with vehicle (UNT) or $1000 \mathrm{nM}$ pacritinib, quizartinib, or ruxolitinib for 16 hours. Phosphorylation status of FLT3, IRAK1, p38, and STAT5 before and after inhibitor treatment are shown. $\alpha$-Tubulin serves as a loading control. Results shown are representative of 3 independent experiments.

Figure 4. Pacritinib treatment showes significant ex vivo efficacy in patient-derived primary AML cells. Agarwal laboratory culture freshly processed patient-derived primary AML cells for 72 hours with graded concentrations of pacritinib, quizartinib, and ruxolitinib from 1.2 $\mathrm{nM}$ to $10,000 \mathrm{nM}$ using a 2-fold drug dilution. Cell viability was measured by colorimetric MTSbased assay in triplicate. (A) Scatter plot showing cell-based median $\mathrm{IC}_{50}$ values. (B) Scatter plot showing cell-based median IC90 values. (C) Scatter plot showing AUC values. (D) Scatter plot showing the correlation between $\mathrm{AUC}$ and $\mathrm{IC}_{50}$ values determined by Agarwal laboratory. (E) Immunoblot analysis of lysates prepared from mononuclear cells derived from 4 independent patients with AML and treated with vehicle (UNT), $200 \mathrm{nM}$ pacritinib or quizartinib as well as $200 \mathrm{nM}$ pacritinib in the presence and absence of $10 \mathrm{ng} / \mathrm{mL}$ IL-1 $\beta$ or LPS for 16 hours. 
Phosphorylation status of IRAK1, p38, and ERK1/2 before and after inhibitor treatment are shown. GAPDH serves as a loading control.

Figure 5. Genetic targeting of IRAK1 or pacritinib treatment leads to a significant reduction in leukemia burden. (A) MOLM-14 cells transduced with the doxycycline (Dox) inducible IRAK1 shRNA were transplanted into NSG mice $\left(2 \times 10^{5}\right.$ cells /mouse $)$ by tail vein injections. 5 days post-engraftment, after confirmation of equal engraftment in peripheral blood $(>1 \%)$, the mice were randomly divided in three groups and treated with vehicle control, or 150 $\mathrm{mg} / \mathrm{kg}$ pacritinib twice daily or $500 \mu \mathrm{g} /$ mouse doxycycline per day by oral gavage. Doxycycline arm of mice were also maintained at the chow containing $625 \mathrm{mg} / \mathrm{kg}$ doxycycline. All the mice were sacrificed 18 days post treatment and MOLM-14 cells engraftment was determined by measuring hCD13/CD33, and GFP-positivity and contribution of murine cells were analyzed by measuring mCD45 and GFP-negetive cells in bone marrow (B) and spleen (C) by flow cytometry. (D) The representative FCAS plots are shown for all the treatment conditions. (E-F) The difference in liver and spleen sizes are shown. The data is represented as mean $\pm \mathrm{SEM}$. ${ }^{*} \mathrm{p} \leq 0.05, * * \mathrm{p} \leq 0.01$, $* * * \mathrm{p} \leq 0.001$. 


\section{Figure 1}

A
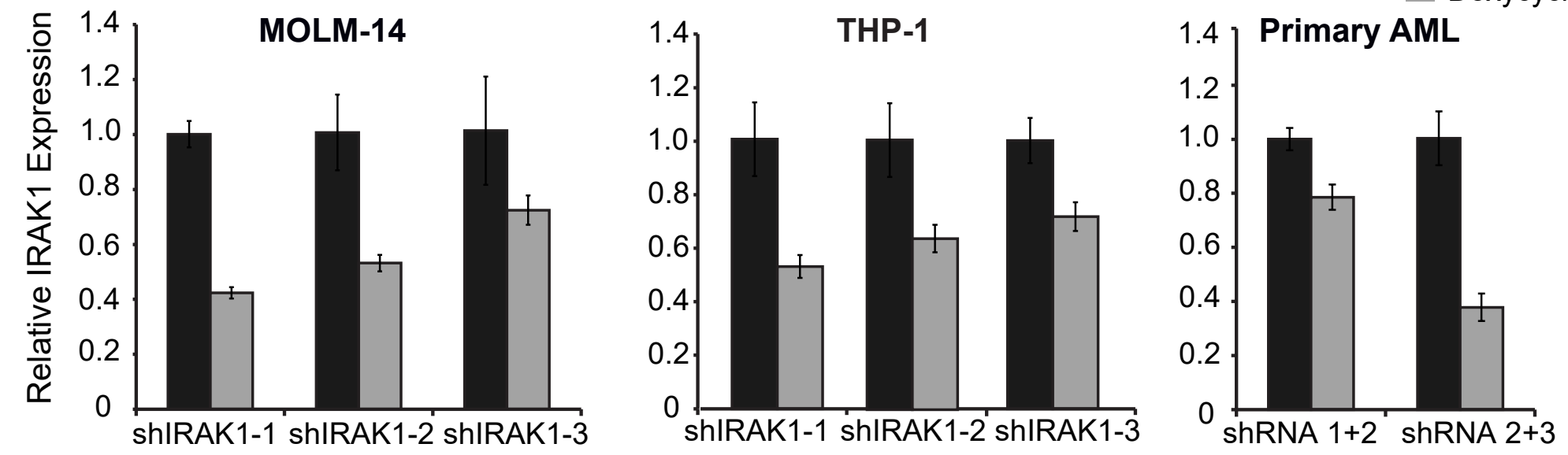

B

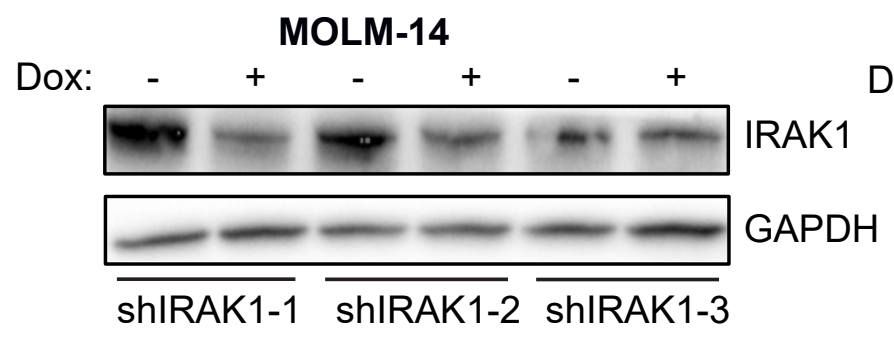

C
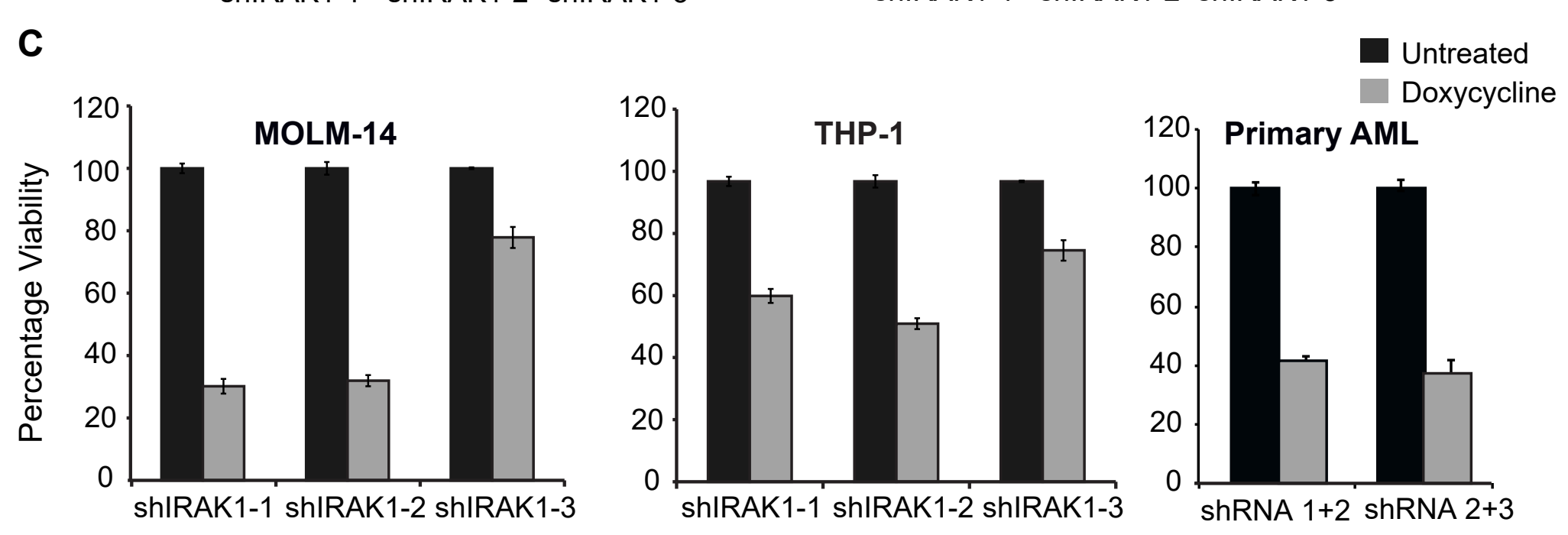

THP1

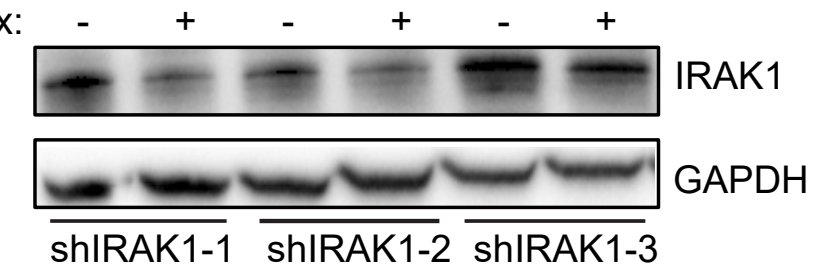


Figure 2

A

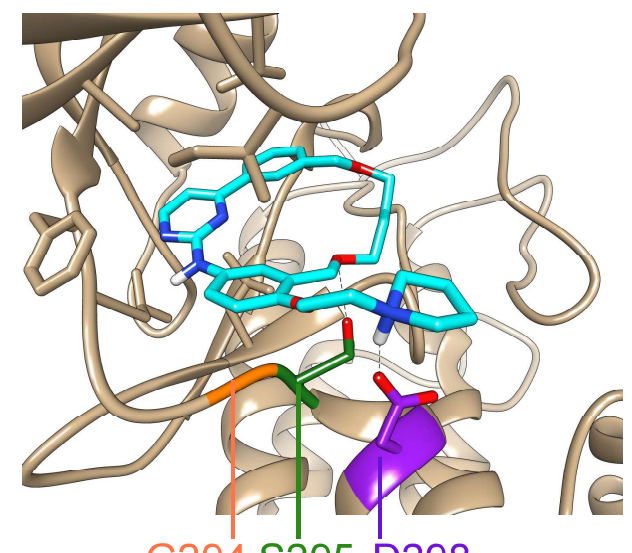

B

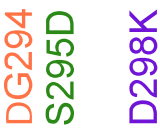

IRAK1 P N I VDFAGYCAQN - G FYCLVYGF LPNGSLEDR L IRAK2 P NV LPVLGFCAAR - - Q F HSF I Y P Y MANG SLQDR L IRAK3 P N I L E LAAY F T ET - - E K F C L I Y P Y MR NGTL F DR L IRAK4 EN LVELLGFS SDG- - DD LCLVYVYMP NG SLLDR L JAK2 DN I VKY K GVC Y S A GR R N L K L I ME Y L P Y G SLRDY L FLT3 EN I V NLLGACTL S - GP I Y L I FEY C C Y GDL LNY L ROS1 PN I LKQLGVCLLN- - EPQY I I LELMEGGDLLTY L CDK2 P N I VKL LDV I HT E - NKLYLVFEFLHQ-DLKKFM

C

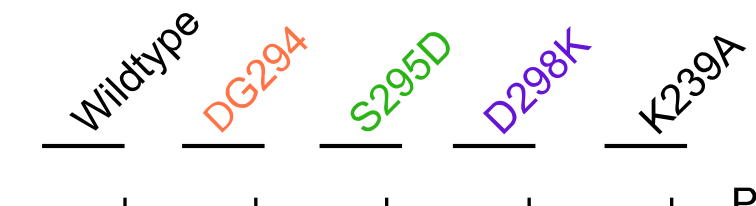

$-+-+\ldots+\ldots+\ldots$ Pacritinib

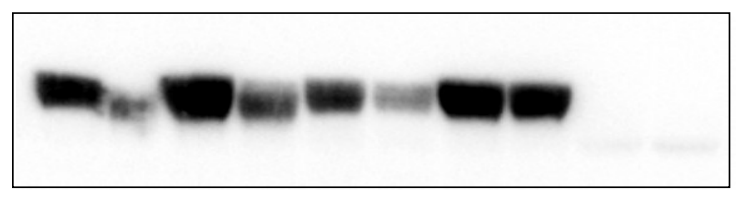
$(1 \mu \mathrm{M})$

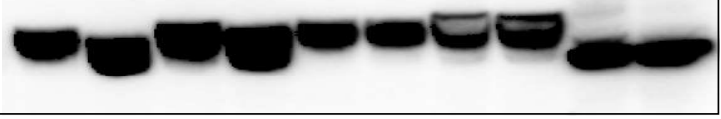

IP: Flag-IRAK1

Flag-IRAK1

p-IRAK1 (Thr 209$)$

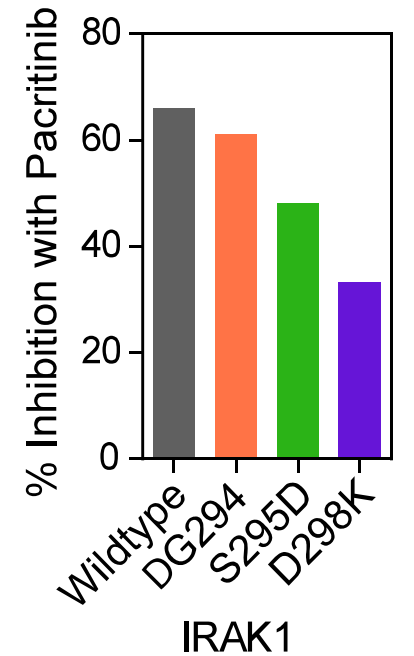


Figure 4
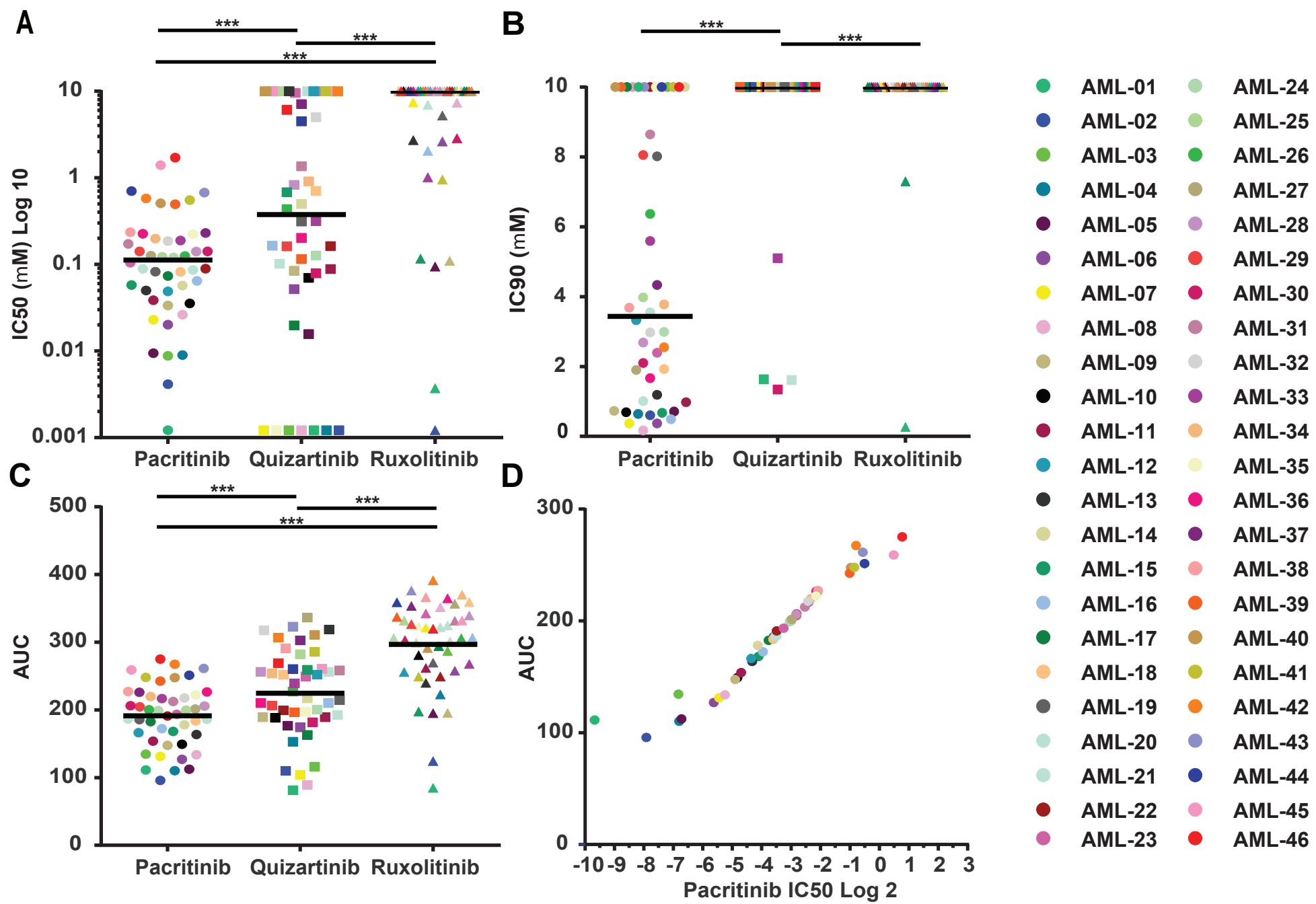

E

AML-36 (FLT3-ITD,CEBPA)

\begin{tabular}{c} 
AML-42 (KRAS,SRSF2) \\
\hline UNT PAC QUIZA
\end{tabular}
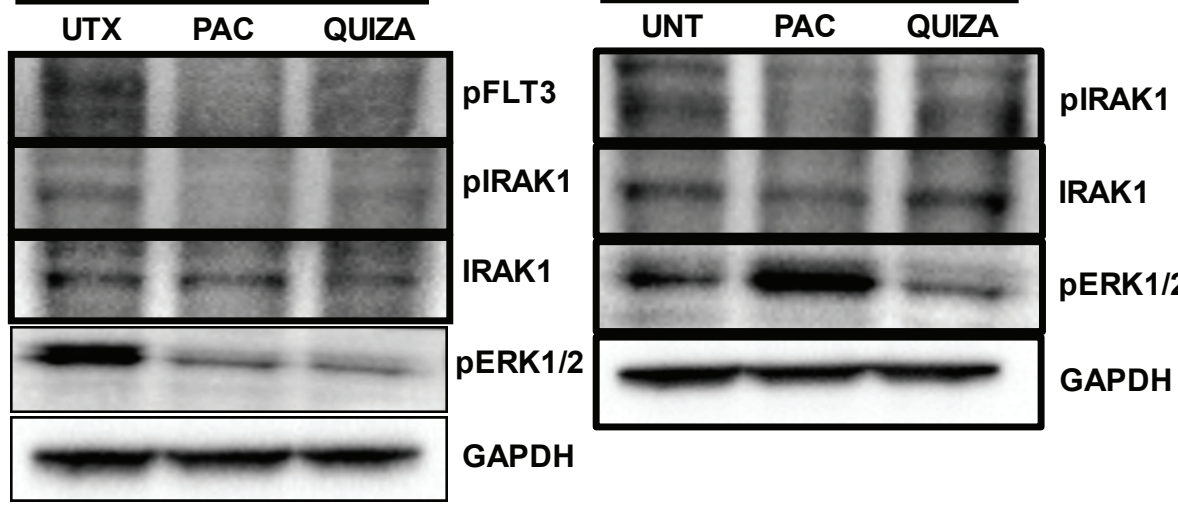

IRAK1

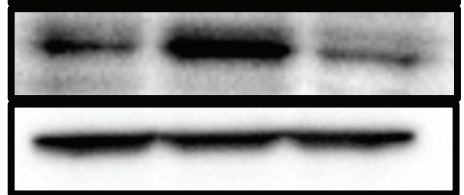

pERK1/2

PERK $1 / 2$

GAPDH

GAPDH

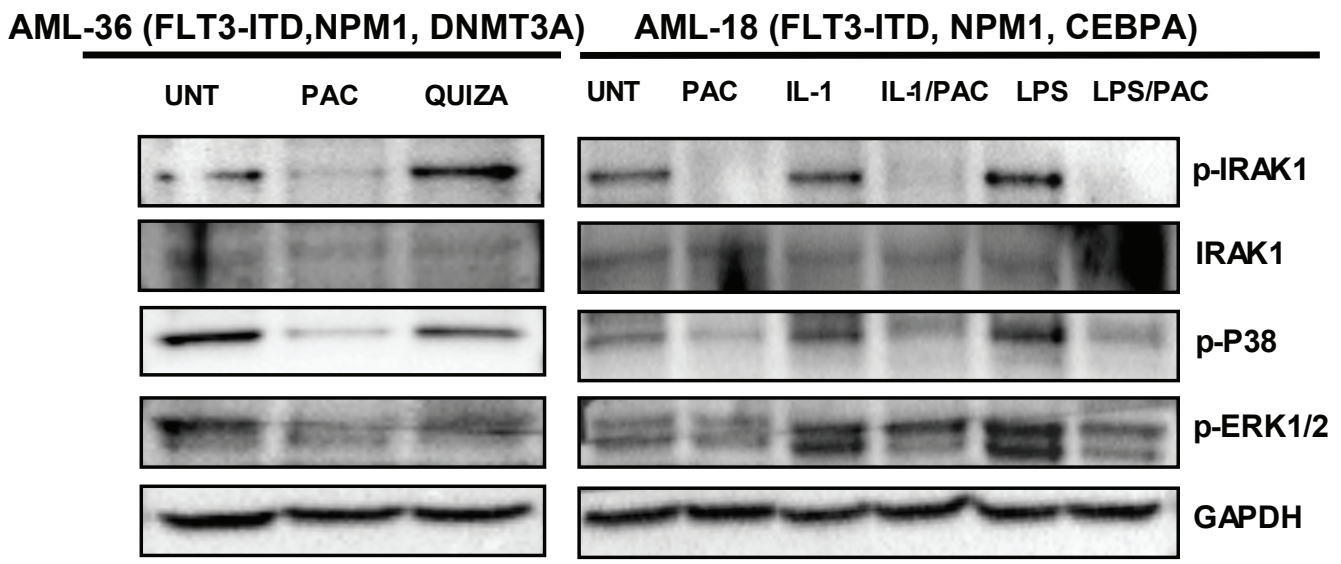




\section{Figure 5}

A

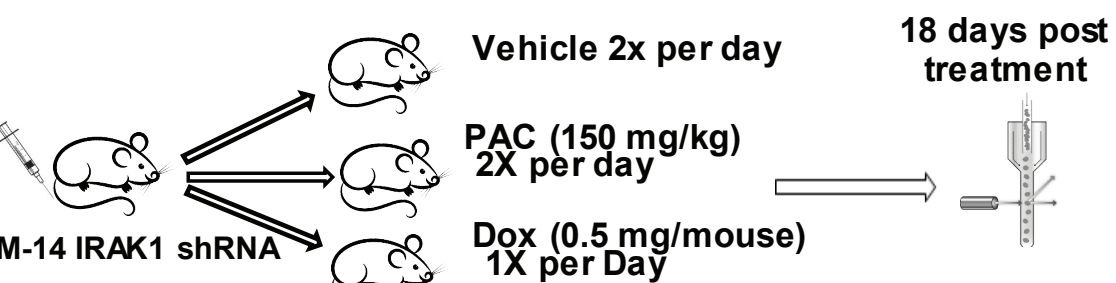

B

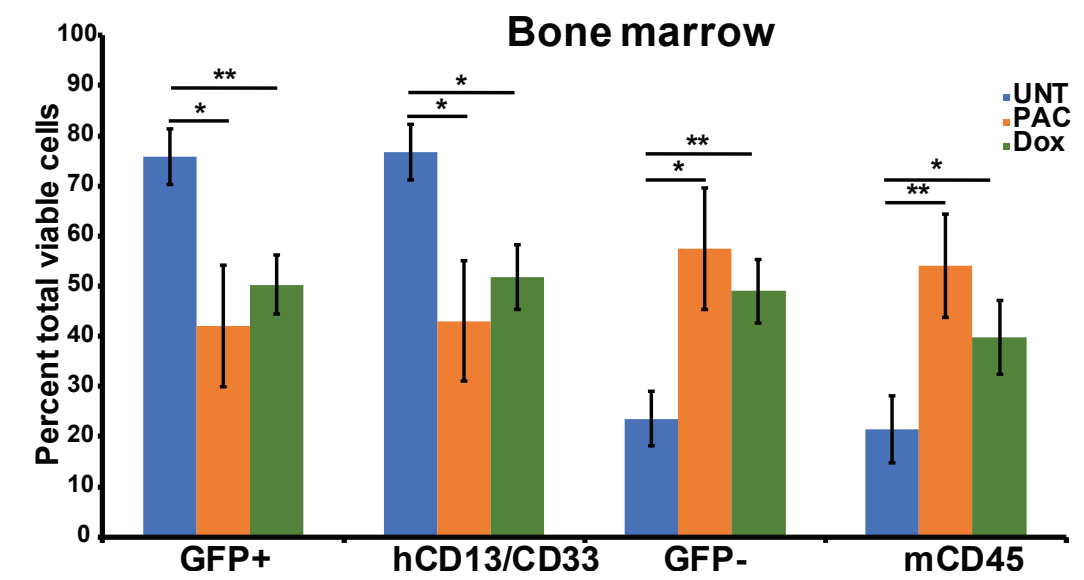

D

\section{Bone marrow}
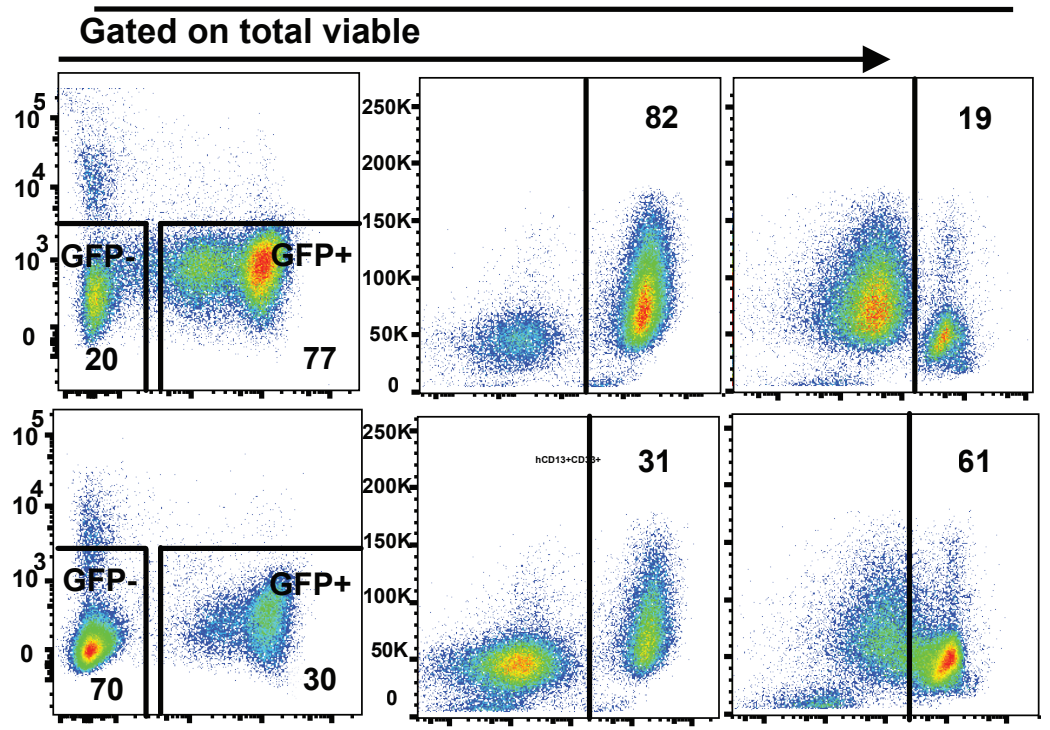


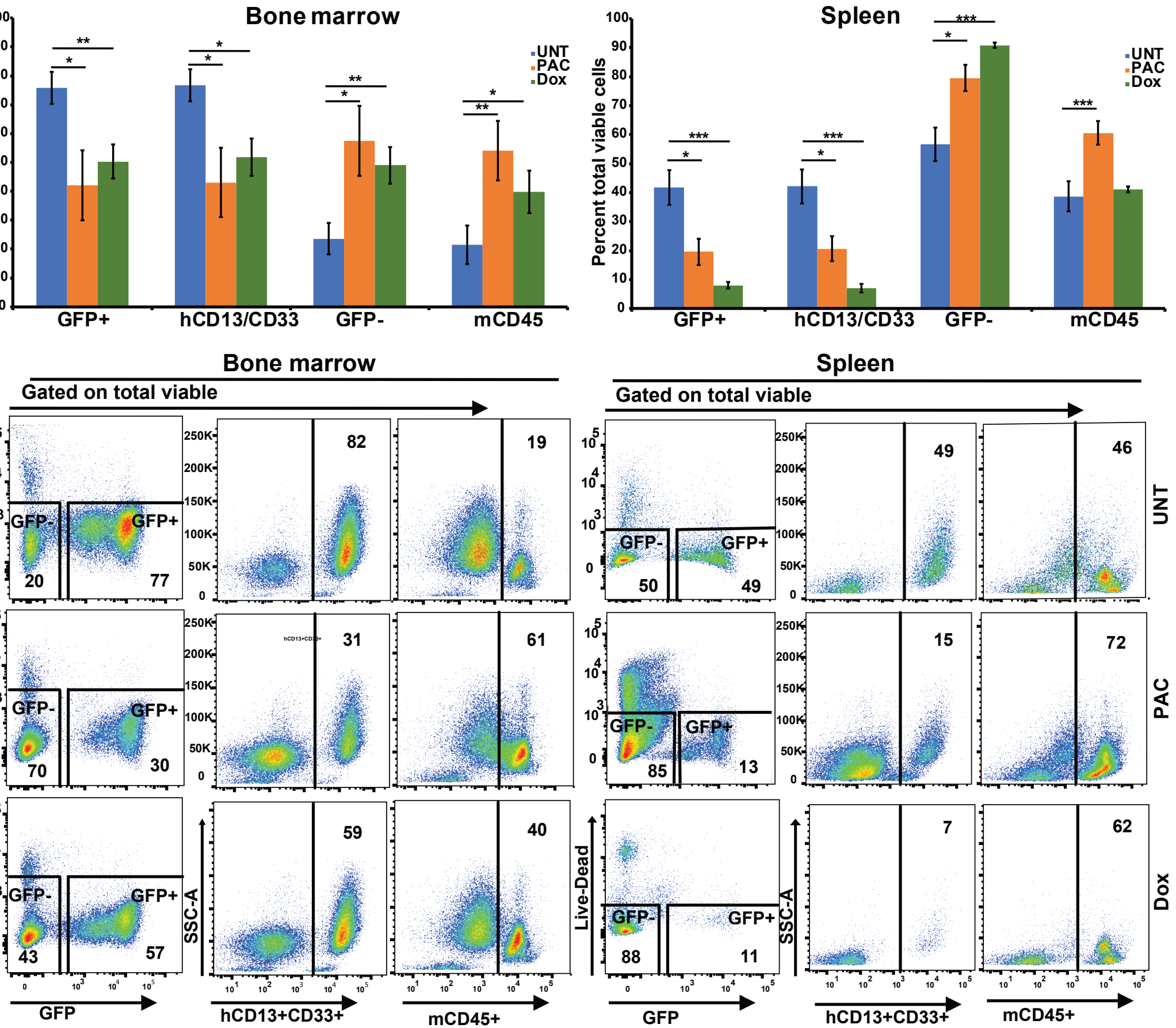

\section{Spleen}
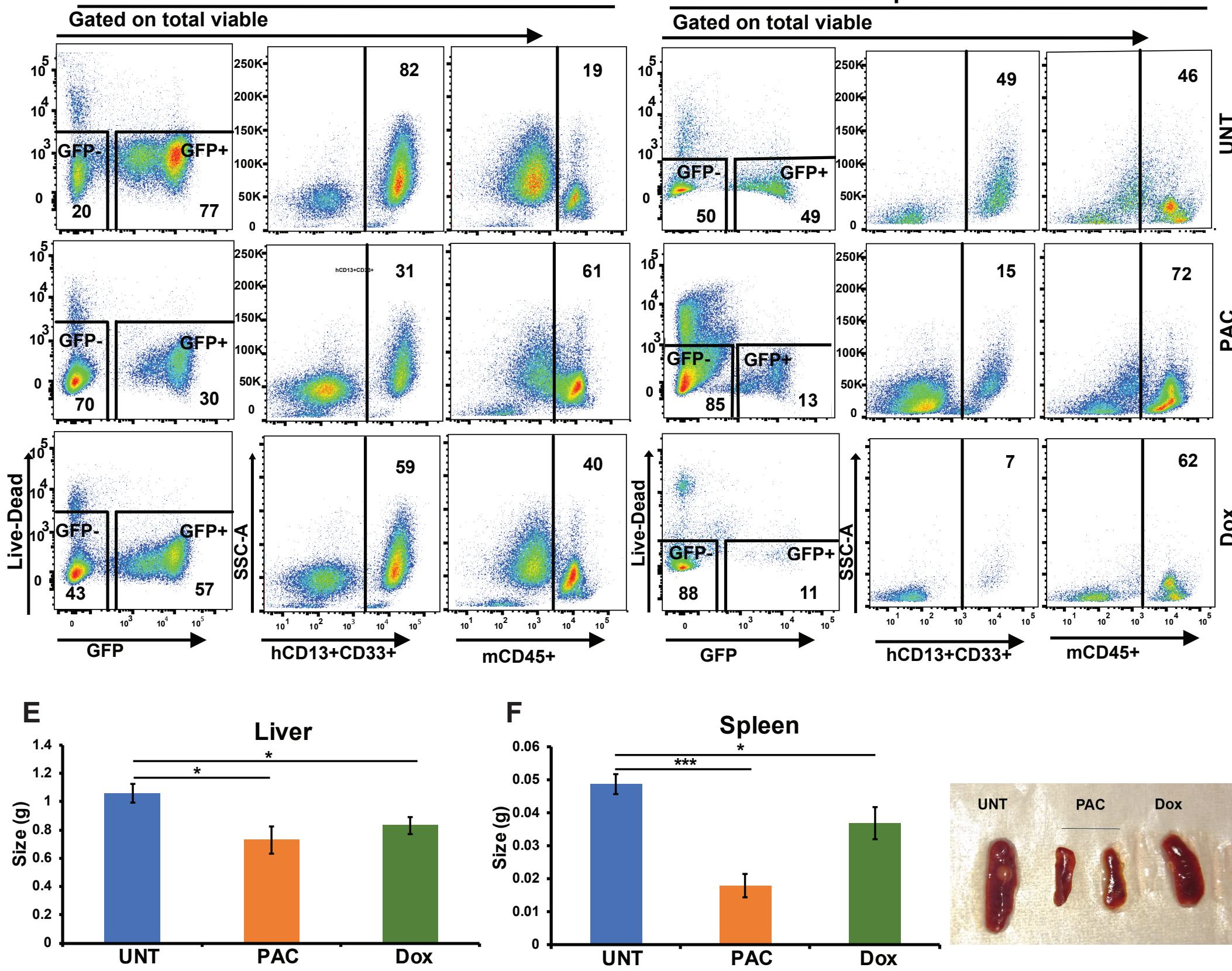


\section{Supplemental Figure 1}
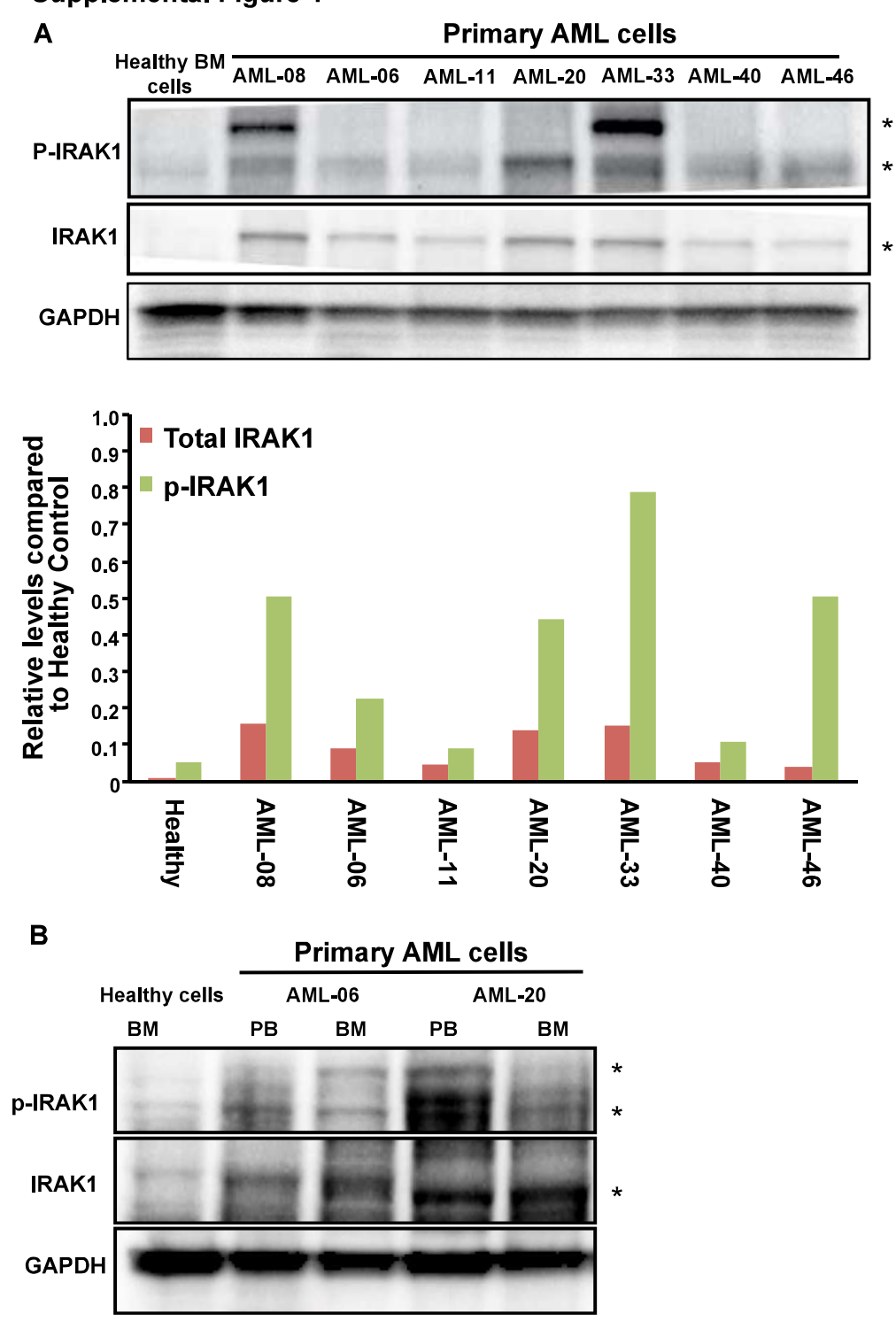

Supplemental Figure 1. IRAK1 is overexpressed in primary AML cells and constitutively

phosphorylated. (A) Cell lysates were prepared from mononuclear cells derived from the bone marrow $(\mathrm{BM})$ or peripheral blood $(\mathrm{PB})$ of healthy individuals and patients with AML. Total IRAK1 and pIRAK1 levels were evaluated by immunoblot analysis. Densitometry analysis was performed to measure IRAK and pIRAK1 levels using GAPDH as the loading control. (B) IRAK1 and pIRAK1 levels in paired BM and PB samples. *Indicates 78-80 Kda pIRAK1 and IRAK1 bands. The larger size band with pIRAK1 antibody might be due to the ubiquitination of IRAK1 as described previously (Rhyasen, G. W. et al Cancer Cell, 2013, Ref: 16) 


\section{Supplemental Figure 2}

MOLM-14

\begin{tabular}{|c|c|c|c|c|c|c|c|c|c|c|c|c|c|c|c|}
\hline UNT & 50 & 200 & 500 & 1000 & 1500 & 2000 & 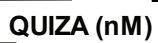 & UNT & 50 & 200 & 500 & 1000 & 1500 & 2000 & Pacritinib (nM) \\
\hline & & & & & & & pFLT3 & & & & 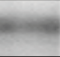 & & & & pFLT3 \\
\hline & & & & & & & pIRAK1 & & & & & & & & pIRAK1 \\
\hline & & $\rightarrow$ & 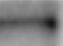 & 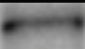 & $8=1$ & 8 & IRAK1 & & & - & & & & 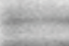 & IRAK1 \\
\hline & 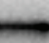 & 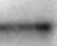 & $m$ & 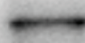 & $+m$ & 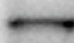 & pP38 & & & & & & & & pP38 \\
\hline & & & & 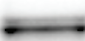 & $\Longrightarrow$ & 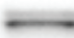 & pERK $1 / 2$ & $=$ & & - & - & - & & & pERK $1 / 2$ \\
\hline & & & & & & & $\mathbf{G}$ & & & & & & & & GAPDH \\
\hline
\end{tabular}

Supplemental Figure 2. Pacritinib inhibits phosphorylation of IRAK1 in AML cells and quizartinib and pacritinib effectively inhibits phosphorylation of FLT3;

however, only pacritinib inhibits phosphorylation of IRAK1. FLT3-ITD positive AML cell line (MOLM-14) was cultured for 16 hours with indicated concentrations of quizartinib and pacritinib. Immunoblot analysis of lysates was performed with indicated antibodies. GAPDH serves as a loading control. Results shown are representative of 3 independent experiments. 
Supplemental Figure 3
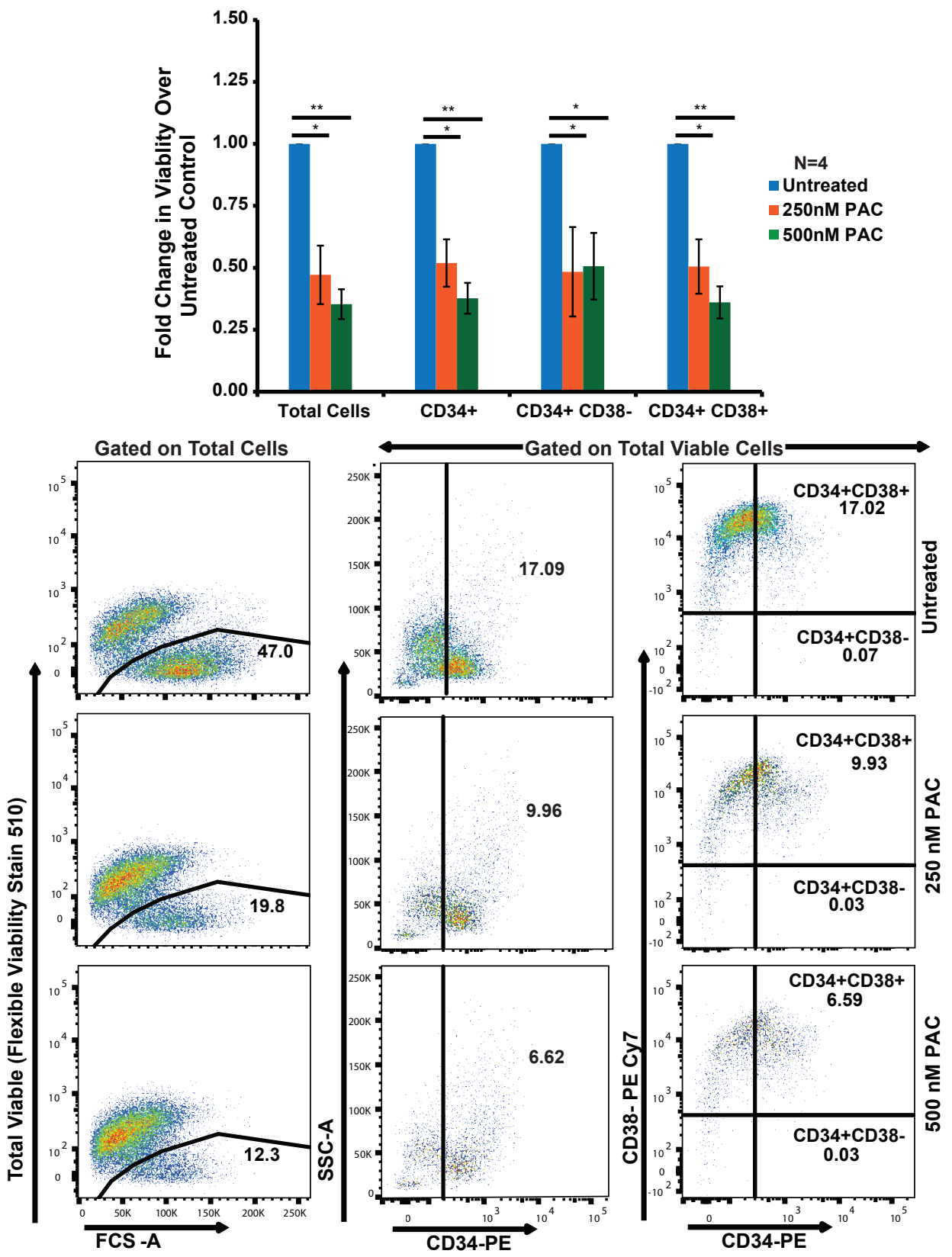

Supplemental Figure 3. Pacritinib treatment reduces the viability of primary AML progenitors ex vivo. Mononuclear cells derived from primary AML samples $(\mathrm{N}=4)$ were cultured in media containing RPMI-1640 supplemented with $20 \%$ FBS and treated with indicated concentrations of pacritinib for 72 hours. Cells were stained with fixable viability stain, CD34, and CD38 surface markers. The data was collected by flow cytometry and analyzed using FlowJo software. The bar graph showed the fold change in viability over untreated control for each subpopulation and is represented as mean \pm SD. The representative plots showing the percentage of total viable, viable CD34+, viable CD34+CD38-, and CD34+CD38+ subpopulations for indicated treatment from one AML sample. 
Supplemental Figure 4
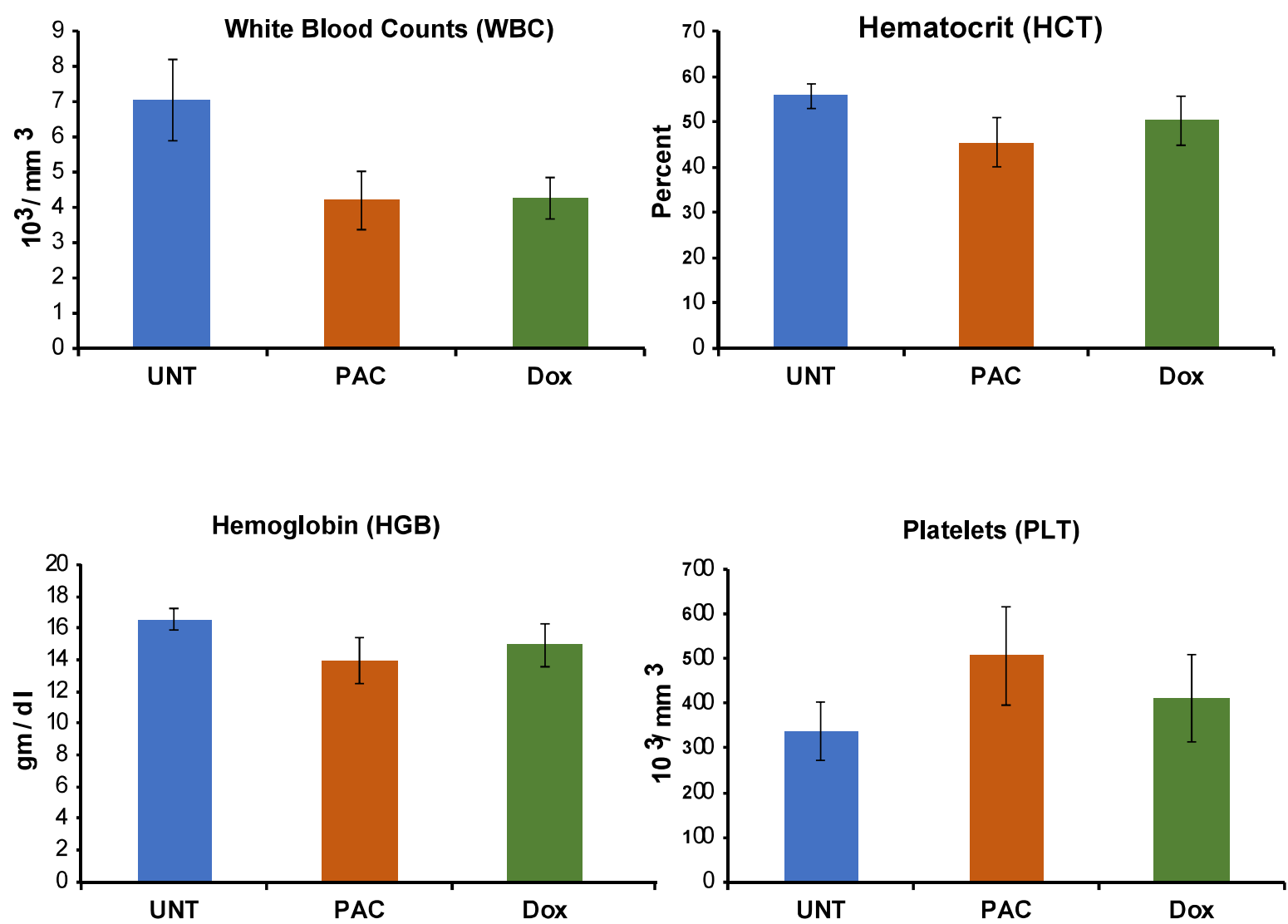

Supplemental Figure 4. The effect of IRAK1 targeting or pacritinib treatment on peripheral blood differentials. MOLM-14 cells transduced with the doxycycline (Dox) inducible IRAK1 shRNA were transplanted into NSG mice (Figure 5). 5 days postengraftment the mice were randomly divided in three groups and treated with vehicle control, or $150 \mathrm{mg} / \mathrm{kg}$ pacritinib or $500 \mu \mathrm{g} /$ mouse doxycycline by oral gavage. All the mice were sacrificed 18 days post treatment and the blood differentials that were measured before the end point are shown. The data is represented as mean \pm SEM. 